\title{
A Novel Ontology Consistent With Acknowledged Standards in Smart Homes
}

\author{
Huansheng Ning, Senior Member, IEEE, Feifei Shi, Tao Zhu, Qingjuan Li, and Liming Chen, Senior Member, IEEE,
}

\begin{abstract}
With the development of Internet of Things, the Smart Home equipped with various sensors and devices has become a hot area attracting global attention and concern. In order to get a better understanding of ambient environments, adding semantics to sensor data plays a significant role. Researchers are attempting to build semantic models in order to satisfy their own requirements, which leads to little reusability between different models. This paper aims to provide a novel ontology which follows publicly acknowledged standards for achieving sensor data semantization in Smart Homes, including modeling sensors, context and activities with semantics. For keeping consistent with current accepted standards, the proposed ontology is based on the Semantic Sensor Network Ontology. In addition, we enrich the ontologies by incorporating spatiotemporal information and user profiles. The ontology is designed using Protégé and a use case is demonstrated to show the great potentiality in daily activity recognition in smart homes.
\end{abstract}

Index Terms-Ontology; Standards; Smart Homes; Internet of Things

\section{INTRODUCTION}

The development of the Internet of Things (IoT) gives birth to many new areas such as Smart Homes (SH), Smart Cities and Smart Transportation. As a product of IoT, The Smart Homes (SH) [1] are becoming more and more prevalent closely related to daily life. Compared with other devices, sensors are superior in diversity, energy consumption and privacy protection, and have become first choices deployed in SH sensing and detecting ambient environments. In order to standardize the descriptions of sensors, the Open Geospatial Consortium (OGC) publishes a suite of specifications about sensors and sensor data models [2] including Observations

H. Ning, F. Shi and Q. Li are with School of Computer and Communication Engineering, University of Science and Technology Beijing, Beijing, 100083, China, e-mail: ninghuansheng@ustb.edu.cn.

H. Ning is also with Beijing Engineering Research Center for Cyberspace Data Analysis and Applications, Beijing, 100083, China.

T. Zhu is with Software School,University of South China, Hengyang, 421001, China.

L. Chen is with School of Computer Science and Informatics, De Montfort University, Leicester, LE1 9BH, UK, e-mail: liming.chen@dmu.ac.uk. and Measurements (O\&M) [3], Sensor Model Language (SensorML) [4] and Sensor Observation Service (SOS) [5]. Considering the practical applications in $\mathrm{SH}$, we focus on the introduction of O\&M and SensorML.

- O\&M provides XML schemas for observations and for features involved in sampling when sensors make observations and measurements.

- SensorML is designed to describe sensor systems and processes in robust and semantically-tied means. The aim is to enhance interoperability at both syntactic level and semantic level thus sensor data can be interpreted and understood better by machines.

For overcoming the heterogeneity [6] brought by various sensors, data semantization plays a significant role. It provides a unified expression architecture which supports high level intelligent applications, including activity recognition, risk detection and decision making. Many studies concentrate on building semantic models in SH related to environment context, sensors and activities. However, most of them ignore the existing standards and a majority of the models are isolated and fail to be reused and extended. An ontology aimed at describing sensors and observations [7] named the SSN ontology was published by the W3C Semantic Sensor Networks Incubator Group (SSN-XG). It covers a large part of OGC standards like O\&M and SensorML but is lack of descriptions of other areas [8]. Therefore, this paper focuses on building semantic models in $\mathrm{SH}$ on the basis of SSN ontology in order to keep in line with existing standards.

Also for providing a comprehensive ontology in $\mathrm{SH}$, new concepts such as spatiotemporal descriptions and user profiles would be incorporated for semantic richness. Current researches related to semantic modeling in assisted living usually ignore the influence of time, position, context as well as preferences and habits of users. However, spatiotemporal semantics provide a better understanding of ambient environment, and user profiles make it possible to confirm the triggers of activities in smart homes. 
The remainder of this paper is organized as follows. Section 2 discusses the current work and researches related to semantic modeling. Section 3 presents the proposed semantic ontology merging with existing standards, spatiotemporal factors and user profiles which is implemented in protégé, and analyzes the advantages of the semantic ontology. Section 4 demonstrates simple examples of activity recognition in smart homes based on the proposed semantic ontology. Finally section 5 gives a conclusion and points out future directions.

\section{RELATED WORK}

Data semantization plays an important role in achieving a better understanding of ambient environments. With the increasing attention on smart homes and assisted living, researchers make a lot of efforts on modeling context, sensors and activities with semantics. Our previous work has made a survey of sensor data semantization in smart homes [8] and this section mainly focuses on current semantic models in relation to spatiotemporal information and user profiles.

In 2009 Chen [9] first proposed the concept of Semantic Smart Homes (SSH). A SSH is composed of seven ontologies including ontologies for the phyical equipments, Activities of Daily Living (ADLs), living spaces, actors, medical information, software components and time. It's a great leap in the progress of semantic developments with spatiotemporal annotations while the semantic models are limited to conditions where there is only single habitant living in the observed area. Ye [10] presents a toplevel ontology model with combination of domain knowledge. The semantic model pays the emphasis on describing information at different levels of granularity. It defines four relationships between ground activities and abstract activities-equal to, finer-grained, conflicting and overlapping. However, these semantic models are lack of temporal semantics. Augusto [11] adopts temporal reasoning to detect complex events in smart homes and the models are demonstrated to provide instant supports in hazardous situations. Jakkula [12] also builds semantic models in smart homes with adding temporal analysis. In addition, Dey [13] gives a perfect use case with adding spatiotemporal annotations to an energy sensor ontology, in which spatial and temporal concepts are incorporated in order to support related analysis and queries. Besides application areas of smart homes and assisted living, spatial and temporal semantics are mostly added into ontologies in Geospatial Semantic Web (GSW) [14]. Ren [15] presents a spatiotemporal ontology representing the geographical information which consists of a spatial and a temporal model. Hancock [16] implements an ontology in Geographic Information System (GIS) with spatial and temporal
TABLE I

Comparison Between Different Semantic Models.

\begin{tabular}{c|c|c|c|c}
\hline Name & Temporal & Spatial & $\begin{array}{c}\text { User } \\
\text { Profiles }\end{array}$ & Application Areas \\
\hline Chen [9] & $\mathrm{Y}$ & $\mathrm{Y}$ & $\mathrm{N}$ & Smart Homes \\
\hline Ye [10] & $\mathrm{N}$ & $\mathrm{Y}$ & $\mathrm{N}$ & Smart Homes \\
\hline Augusto [11] & $\mathrm{Y}$ & $\mathrm{Y}$ & $\mathrm{N}$ & Smart Homes \\
\hline Jakkula [12] & $\mathrm{Y}$ & $\mathrm{Y}$ & $\mathrm{N}$ & Smart Homes \\
\hline Dey [13] & $\mathrm{Y}$ & $\mathrm{Y}$ & $\mathrm{N}$ & Smart Homes \\
\hline Ren [15] & $\mathrm{Y}$ & $\mathrm{Y}$ & $\mathrm{N}$ & GSW \\
\hline Hancock [16] & $\mathrm{Y}$ & $\mathrm{Y}$ & $\mathrm{N}$ & GIS \\
\hline Wang [17] & $\mathrm{Y}$ & $\mathrm{Y}$ & $\mathrm{Y}$ & Smart Homes \\
\hline Heiyanthuduwage [18] & $\mathrm{N}$ & $\mathrm{N}$ & $\mathrm{Y}$ & Learning \\
\hline Jayakumar [19] & $\mathrm{N}$ & $\mathrm{N}$ & $\mathrm{Y}$ & Internet Searching \\
\hline
\end{tabular}

semantics. They all demonstrate the necessity and feasibility of adding spatial and temporal semantics in ontology modeling.

In addition to semantic factors mentioned above, user profiles are attracting researchers' focus. For getting a better recognition of individualized activities, the relationships between activities and user profiles need to be taken into considerations. Wang [17] proposes an ontology concentrating on the influence of different habits in which a habits library is predefined and with the help of users' habits, it's much more accurate to recognize activities in assisted living. Although users' habits have been merged in the semantic model, it's only assisted for recognizing daily activities rather than confirming the triggers of the activity. Actually adding user profile factors into models in smart homes is not common because of the difficulties to distinguish different habitants and to recognize intersecting activities. Instead, user profiles are widely adopted in other application areas. Heiyanthuduwage [18] adds user profiles in a learning ontology in order to enhance the accessibility of resources, which mainly refers to Interests of users and Features of Objects. Jayakumar [19] builds an internet searching ontology based on user profiles for providing much more accurate search results. User profiles like users' basic information and education details are concentrated in this semantic model. A simple comparison has been made between different semantic models in terms of spatiotemporal semantics, user profiles and the application areas. The results are shown in Table I.

Although researchers are committed to building semantic models in assisted living, there is no model which keeps in line with existing standards and covers all semantic factors in smart homes. This paper presents a novel ontology merging with current standards such as O\&M and SensorML, and incorporating spatiotemporal semantics as well as user profiles when creating semantic models. A detailed introduction would be given in next section. 


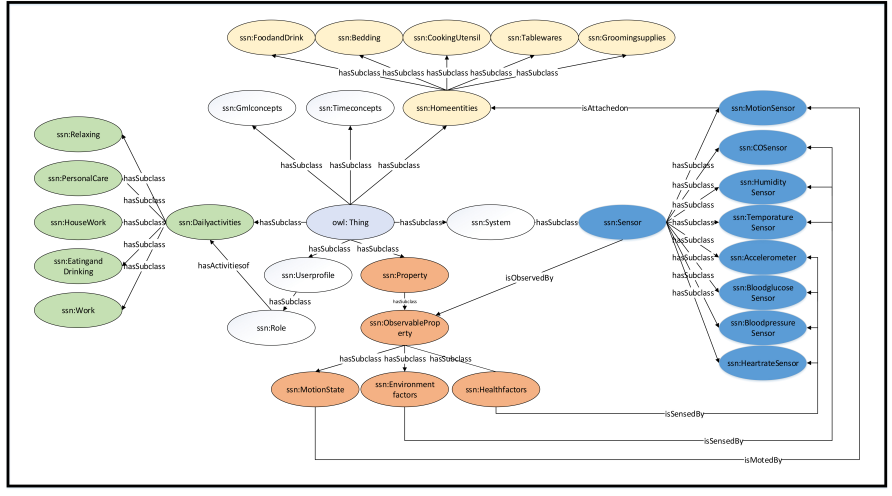

Fig. 1. The Typical Ontology Concepts in Smart Homes.

\section{The Implementation of the Semantic Ontology}

This section gives a detailed introduction to the proposed semantic ontology with spatiotemporal annotations and user profiles semantics, which is expanded from the acknowledged ontology The SSN ontology for in accord with current publicly accepted sensor standards.

\section{A. $O \& M$ and SensorML Alignment}

As Compton [7] points out that the SSN ontology adheres to current standards of sensors, especially O\&M and SensorML, the main template of sensor and observation descriptions in our proposed ontology is based on the SSN ontology for keeping consistent with existing standards of sensors. In addition, some extensions and improvements are made such as the detailed types of sensors and the relationships between sensors and observable property. The typical concepts involved in smart homes are shown in Figure 1, in which sensors and observations are represented in blue and orange ovals separately.

Among all potential applications in assisted living, activity recognition and risk detection are most popular thus the designing and expanding of the ontology are around these two applications. In order to provide a better description of sensors (ssn:Sensor), eight types of sensors are mentioned, that is, humidity, $\mathrm{CO}$ and temperature sensors for environmental factors, heart rate, blood pressure, blood glucose sensors and accelerometers for healthy factors, and motion sensors for sensing any contact or motion events. Compared with other sensors, motion sensors are distinct because their measurements are ssn:On when motion events are triggered instead of detailed values. Therefore, the original relationship ssn:isObservedBy between sensors and observable property
(ssn:ObservableProperty) is refined to ssn:isMotedBy for motion sensors and ssn:isSensedBy for others. Meanwhile the observable property is mapped to corresponding measurements such as factors for health, factors for environment and motion states.

One drawback of the SSN ontology is that it's lack of temporal and spatial information related to sensors and sensor data. There is only placeholder in ssn:Observation for recording spatiotemporal information with no more detailed introductions, and it ignores the influences of different habits of users. Therefore, besides original concepts in the SSN ontology, spatiotemporal annotations and user profiles are added in our proposed semantic models.

\section{B. Spatiotemporal Semantics}

Spatiotemporal semantics in relation to sensor data are more and more crucial in real applications for satisfying relevant queries. It's obvious spatiotemporal information provides a better understanding of the ambient environments. In assisted living, it's significant to identify the location and time of triggered sensor events in order to recognize exactly what is happening in the observed area. For enhancing the stability, usability and credibility of semantic models, a semantic ontology with spatial and temporal components is built on the basis of current researches.

1) Spatial Semantics: The Geography Markup Language (GML) [20] is a standard expression format defined by OGC for expressing spatial features, including Coordinate Reference System (CRS), geometries, coverage, coordinates and so forth. As a publicly accepted modeling language for spatial information, GML has been adopted as a template in many researches for spatial semantic richness. Therefore, this paper makes extensions based on GML. The spatial concepts are created by protégé as shown in Figure 2 (a). The semantic ontology mainly focuses on the concept of location (ssn:location), which has subclasses such as ssn:Studyroom, ssn:Bathroom, ssn:Bedroom, ssn:Kitchen and ssn:Livingroom. A relationship between location, daily activities (ssn:Dailyactivities) and home entities (ssn:Homeentities) is defined as ssn:locatedin, which expresses the spatial information of entities in smart homes in order to support geographical queries. Although coordinates and CRS provide a more rigorous representation for spatial information, only definitions are given considering the real applications in smart homes with no more explicit explanations. When the application scope is extended to the community, these concepts such as ssn:Coordinates and ssn:hasCoordinatesof can be easily expanded for better use.

2) Temporal Semantics: In addition to spatial semantics, temporal information holds an important role in creating a comprehensive knowledge representation for smart homes. Regarding temporal 


\begin{tabular}{|c|c|}
\hline \begin{tabular}{|l} 
Gmlconcepts \\
Coordinates \\
Directions \\
Location \\
Studyroom \\
Bathroom \\
Bedroom \\
Kitchen \\
Livingroom
\end{tabular} & \begin{tabular}{|c|} 
Timeconcepts \\
TimeGranularity \\
Day \\
Hour \\
Minute \\
Month \\
Second \\
Week \\
Year \\
TIMEX3 \\
TemporalInterval \\
TinalInstant \\
Initialinstant \\
Durations \\
Time
\end{tabular} \\
\hline (a) The Spatial Concepts & (b) The Temporal Concepts \\
\hline
\end{tabular}

Fig. 2. The Spatial and Temporal concepts.

semantics, TimeML [21] is a robust specification language for events and temporal expressions. As a standard expression format for temporal information, TimeML is aimed at tackling problems related to time stamping, ordering and reasoning about sensor events. In our semantic ontology, temporal concepts are built based on TimeML.

Temporal information provides the possibilities to understand the ambient environment better. When sensor events are triggered in smart homes, sensor data come with time stamps indicating the time point that the event is anchored. In our proposed semantic ontology, temporal concepts mainly consist of time ganularity (ssn:TimeGranularity) and TIMEX3 (ssn:TIMEX3) shown in Figure 2 (b). ssn:Time records the time stamps of events anchored by sensors, and it has granularity such as ssn:Day, ssn:Hour, ssn:Minute and so forth. Besides, daily activities are composed of sensor events with anchored time thus the time interval (ssn:TemporalInterval) composed of ssn:InitialInstant and ssn:FinalInstant of activities can be inferred.

\section{User Profiles Semantics}

Users play important roles in triggering different events or activities in smart homes. Especially under environments of multiple residents, users' profiles such as habits, preferences as well as operation sequences of using devices have tremendous influences on activity recognition. In our proposed ontology, user profiles such as roles, preferences and basic information are modeled and the key concepts are shown in Figure 3.

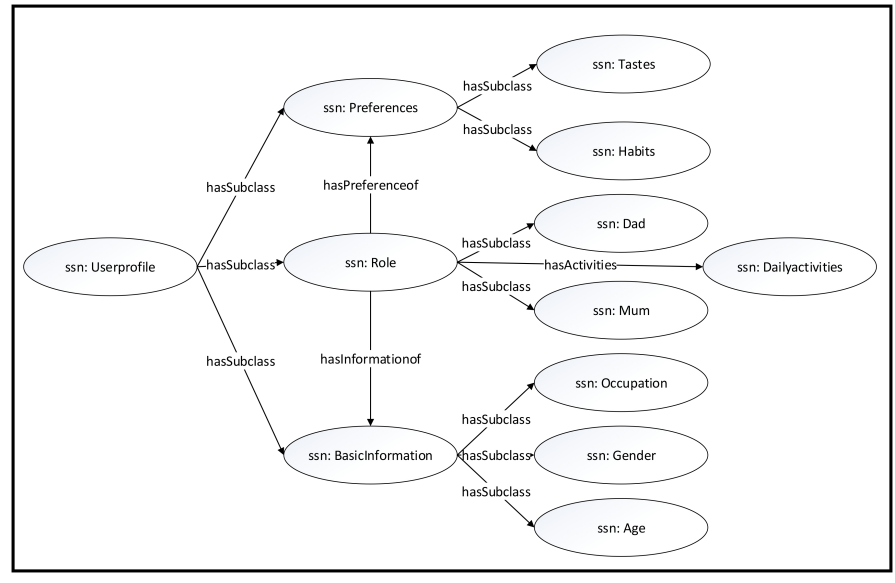

Fig. 3. The Concepts of User Profiles.

In our work, typical roles are defined as ssn:Mum and ssn:Dad, which are representative users in smart homes. For simplicity, a premise that ssn:Mum is a house wife engaged in caring family whereas ssn:Dad needs to work in order to support the whole family is given at first. Taking cooking (ssn:Cooking) and work (ssn:Work) as examples, it's a normal phenomenon ssn:Dad may work overtime at home and ssn:Mum is used to cooking at a certain point of time. With the help of the transcendental knowledge, we can infer the trigger is ssn:Mum instead of ssn:Dad when ssn:cooking is detected in the morning.

Besides, the preferences and habits of users can be regarded as part of user profiles. In Chinese typical family, preferences and habits are worth being focused. For example, some people like coffee while others are fascinated by tea. Some people are used to sleeping early while others prefer to stay up late. In our proposed model, preferences and habits are described in Object properties to a large extent. Sub properties of owl:topObjectProperty are created such as ssn:hasHabitsof (which includes properties like ssn:hasSleepingTimeof, ssn:hasCookingTimeof and so forth) and ssn:hasPreferencesof (which pays attention to users' tastes preferences, interests preferences and so on). Based on the predefined knowledge base of user profiles, it's much more accurate to confirm triggers of daily activities in smart homes especially under environments of multiple habitants.

\section{Advantages Analysis}

Compared with current semantic models related to smart homes, the proposed ontology incorporates existing publicly accepted standards of sensors, spatiotemporal information as well as user profiles. 
It provides a novel ontology for sensor data semantization in assised living and enhances the standardization, generality, reusability and semantic richness to a large degree.

- Standardization. Considering the research status of data semantization in assisted living, there are no semantic models incorporating existing standards. In this paper, a semantic ontology is proposed based on the SSN ontology which has covered publicly acknowledged standards related to sensors. Home entities, daily activities, spatiotemporal concepts and user profiles are established by extending the SSN ontology. The semantic models have integrated with available standards and prove to achieve a standard representation in assisted living.

- Generality. In order to provide a general approach to data semantization in smart homes, the design of concepts in the proposed ontology covers almost all concepts involved in existing models. With adding semantic labels and markups to general concepts, the proposed semantic ontology has high generality.

- Reusability. In this paper, the proposed semantic ontology is established aimed at typical family in China, thus the concepts involved in the model are more in line with China's family environments, such as the types of sensors, home entities, activities and family roles. All concepts in the ontology are able to be reused in similar home environments.

- Semantic Richness. Semantics make it possible for machines to better integrate, interpret and understand sensor data. The more the semantics, the easier it's to understand the ambient environments. In this semantic ontology, spatiotemporal information and user profiles annotations provide more semantic richness, which have greater potentiality to better comprehend environment context and daily activities in smart homes.

\section{ApPliCATION}

In the proposed semantic model, publicly accepted standards, spatiotemporal concepts and user profiles have been incorporated which are able to support relevant queries, reasoning and analysis. This section presents a demonstration of daily activity recognition in smart homes. We employ Eclipse and Jena as experimental tools and develop reasonable rules in order to make inference and recognition.

In the experiment, ssn:Cooking and ssn:Sleeping are selected as representative daily activities under the environment of multiple habitants. The aim is to prove that with the help of spatiotemporal concepts and user profiles, it's much more accurate when recognizing activities in assisted living.
TABLE II

The Rules Related to ACtivities.

\begin{tabular}{c|c}
\hline Activities & Rules \\
\hline \multirow{3}{*}{ ssn:Sleeping } & (?x AsistiveLiving:hasTriggerState ?y) \\
& (?x AsistiveLiving:locatedIn ?z) \\
& (?z rdf:type AsistiveLiving:Bedroom) \\
& (?z AsistiveLiving:isOwnedby ?u) \\
& $\rightarrow$ (?u AsistiveLiving:hasActivities AsistiveLiving:Sleeping) \\
\hline \multirow{3}{*}{ ssn:Cooking } & (?x rdf:type AsistiveLiving:CookingUtensil) \\
& (?x AsistiveLiving:hasTriggerState ?y) \\
& (?y rdf:type AsistiveLiving:On) \\
& (?x AsistiveLiving:locatedIn ?z) \\
& $\rightarrow$ (AsistiveLiving:Cooking AsistiveLiving:locatedIn ?z) \\
\hline
\end{tabular}

Fig. 4. The Experimental Results.

We assume that there are ssn:Mum and ssn:Dad in the smart homes, and an instance of ssn:Mum named Muml has user profiles shown as follows.

- Muml isOwnerof Bedroom1.

- Mum1 hasCookingTimeof 7:00:00.

Key Rules related to ssn:Cooking and ssn:Sleeping are presented in Table II. Inferences based on rules and sensor events are demonstrated and the experimental results are shown in Figure 4. It tells that Muml has the great possibility of sleeping at Bedroom1 around at 21:00:00 and cooking at 7:00:00. Compared with other models, the proposed ontology in this paper has the ability to infer the triggers of activities with the help of user profiles when recognizing activities. It proves great potentiality in daily activities recognition especially faced with environments with multiple users. 


\section{CONCLUSion ANd Future Work}

In this paper, a novel ontology of sensor data semantization in smart homes is proposed, which incorporates existing standards related to sensors and merges with spatiotemporal concepts as well as user profiles. Firstly, the proposed ontology is a novel method of modeling semantics in smart homes. It keeps in line with publicly accepted standards related to sensors and covers almost all concepts involved in smart homes. And it outstands other models in semantic richness by adding spatiotemporal annotations and user profiles, which helps a lot in comprehending the ambient environments. Also the generality, reusability and interoperability have been highly enhanced in the proposed semantic models. Users can make extensions on the basis of this ontology and define own rules in order to perform high-level tasks, such as activities recognition, risk detection and consistency check.

However, the user profiles in the proposed semantic model are predefined on the basis of transcendental knowledge. In the future, a mechanism of automatic learning of user profiles should be developed in order to update user profiles automatically. In addition, quantized mapping weights describing the relationships between users profiles and activities may be designed and learned by deep learning algorithms. More experiments would be made in order to prove the applicability of the proposed standard ontology for sensor data semantization in assisted living.

Acknowledgments:The work was supported by the National Natural Science Foundation of China (61471035).

\section{REFERENCES}

[1] M. Chan, C. Escriba, and E. Campo, "A review of smart homes-present state and future challenges," Computer Methods \& Programs in Biomedicine, vol. 91, no. 1, pp. 55-81, 2008.

[2] A. Sheth, C. Henson, and S. S. Sahoo, Semantic Sensor Web. IEEE Educational Activities Department, 2008.

[3] "Observations and measurements," Available online: http://www. opengeospatial.org/standards/om(accessed on 25 March 2018).

[4] "Sensor model language (sensorml)," Available online: http://www. opengeospatial.org/standards/sensorml(accessed on 25 March 2018).

[5] "Sensor observation service," Available online: http://www.opengeospatial.org/ standards/sos(accessed on 25 March 2018).

[6] P. Barnaghi, W. Wang, C. Henson, and K. Taylor, "Semantics for the internet of things: early progress and back to the future," International Journal on Semantic Web \& Information Systems, vol. 8, no. 1, pp. 1-21, 2012.

[7] M. Compton, P. Barnaghi, L. Bermudez, R. Garcła-Castro, O. Corcho, S. Cox, J. Graybeal, M. Hauswirth, C. Henson, and A. Herzog, "The ssn ontology of the w3c semantic sensor network incubator group," Web Semantics Science Services \& Agents on the World Wide Web, vol. 17, no. 4, pp. 25-32, 2012.

[8] F. Shi, Q. Li, T. Zhu, and H. Ning, "A survey of data semantization in internet of things:," Sensors, vol. 18, no. 1, p. 313, 2018.
[9] L. Chen, C. Nugent, M. Mulvenna, D. Finlay, and X. Hong, Semantic Smart Homes: Towards Knowledge Rich Assisted Living Environments. Springer Berlin Heidelberg, 2009.

[10] J. Ye, G. Stevenson, and S. Dobson, "A top-level ontology for smart environments," Pervasive \& Mobile Computing, vol. 7, no. 3, pp. 359-378, 2011.

[11] J. C. Augusto and C. D. Nugent, "The use of temporal reasoning and management of complex events in smart homes," in Eureopean Conference on Artificial Intelligence, Ecai'2004, Including Prestigious Applicants of Intelligent Systems, Pais 2004, Valencia, Spain, August, 2004, pp. 778-782.

[12] V. Jakkula, A. Crandall, and D. Cook, "Knowledge discovery in entity based smart environment resident data using temporal relation based data mining," pp. 625-630, 102007.

[13] S. Dey and R. Dasgupta, "Sensor knowledge representation with spatiotemporal annotation: An energy sensor ontology use case," in IEEE International Conference on Pervasive Computing and Communications Workshops, 2014, pp. $455-459$.

[14] C. Zhang, T. Zhao, and W. Li, Geospatial semantic web. Springer, 2015.

[15] J. Ren, X. Wang, X. Lu, and W. Song, "An approach to representing spatiotemporal ontology in the geospatial semantic web," in International Conference on Natural Computation, Fuzzy Systems and Knowledge Discovery, 2016, pp. 2093-2097.

[16] H. Hancock, "Spatio-temporal ontology and its application in geographic information system," in International Conference on Machine Learning and Cybernetics, 2009, pp. 1487-1492.

[17] P. Wang, H. Luo, and Y. Sun, "A habit-based swrl generation and reasoning approach in smart home," in IEEE International Conference on Parallel and Distributed Systems, 2015, pp. 770-775.

[18] S. R. Heiyanthuduwage, R. Schwitter, and M. A. Orgun, "A learning ontology with metadata and user profiles for enhancing accessibility of resources," in E-Learning, E-Management and E-Services, 2017.

[19] P. Jayakumar and P. Shobana, "Creating ontology based user profile for searching web information," in International Conference on Information Communication and Embedded Systems, 2015, pp. 1-6.

[20] “Geography markup language," Available online: https://en.wikipedia.org/wiki/ Geography_Markup_Language(accessed on 25 March 2018).

[21] "Timeml," Available online: https://en.wikipedia.org/wiki/TimeML(accessed on 25 March 2018). 